Seta, J. J., Seta, C. E. \& McElroy, T. (2006). Better Than Better-Than-Average (or Not): Elevated and Depressed Self-evaluations Following Unfavorable Social Comparisons. Self and Identity, 5: 51-72. Published by Taylor \& Francis (ISSN: 1529-8868). DOI: 10.1080/15298860500380551

\title{
Better Than Better-Than-Average (or Not): Elevated and Depressed Self-evaluations Following Unfavorable Social Comparisons
}

\author{
John J. Seta, Catherine E. Seta, and Todd McElroy
}

\begin{abstract}
Two experiments were designed to investigate perceivers' self-evaluations when they received objectively positive above-average performance feedback but were told about another coactor who performed either moderately or much better than the participant. Results indicated that participants responded negatively to this comparison information even though they received better-than-average performance feedback. Participants were given the opportunity to evaluate themselves relative to another coactor who was described as performing at an average level. When the negative implications of the unfavorable social comparisons were relatively mild, both low and high self-esteem participants raised their self-evaluations vis-a 'vis the inferior coactor who performed at an average level on the task. However, when the upward comparisons were especially unfavorable (i.e., when there was a large discrepancy between the performance level of the participant and the coactor-the comparison target), only high self-esteem participants raised their self-evaluations. Results provided evidence for active compensation and relatively passive spreading activation, supporting a schema-maintenance through compensation model (e.g., Seta \& Seta, 1982, 1993; Seta, Seta, \& McElroy, 2003).
\end{abstract}


As noted by Festinger over fifty years ago, the social environment provides us with a rich source of social comparison information that is helpful for self-knowledge in the absence of more objective forms of information (Festinger, 1954). Although this literature is too vast to adequately review in this paper, the research that was generated from his classic social comparison theory also has made it clear that social comparisons can be painful and can represent a threat to our ego or self-concept (see Collins, 1996; Suls \& Wills, 1991). In this paper, we explored how individuals responded to information that they had performed at a level that was above average (i.e., received objectively positive feedback) but also were told that another participant performed either one rank (mild threat) or two ranks (severe threat) above them. A competitive context was induced in which the participants expected to meet with the experimenter to discuss differences in participants' performance levels. These participants also were given information about a performer who had performed at an average level, putting this performer in a relatively inferior social position vis-a' -vis the participant. Thus, our procedures created a social hierarchy of performance comparison information in a competitive context.

Control conditions also were included in which participants received either no information about superior others (Experiment 1) or received information about another performer who performed similarly to the participant (Experiment 2). We asked participants to make self-evaluations of their ability levels relative to the average-other. Based upon the view that participants would respond to these upward comparisons in a relatively negative way, we expected them to be motivated to compensate for these social comparison threats by raising their self-evaluations in relation to the average performer. We also included measurements of participants' self-esteem levels in this research. We expected levels of self-esteem to interact with the social comparison threat conditions such that both high and low self-esteem participants would be able to compensate for mild social comparison threats but that low self-esteem participants would be unable to compensate for relatively severe social comparison threats. In contrast, we expected high self-esteem participants to be able to compensate for relatively severe levels of threat by raising their selfevaluations. Our reasoning for these predictions is discussed below.

We used a schema-maintenance through compensation model (e.g., Seta \& Seta, 1982; Seta, Seta, \& Erber, 1993; Seta, Seta, \& McElroy, 2003) as a theoretical backdrop for this research. Although this model has not been applied to research in social comparison, it is applicable to this domain because unfavorable social comparisons can represent events that are inconsistent with self-related schema. For example, unfavorable upward social comparisons can provide information that one is not meeting goals for self-improvement, or can create negative affective reactions that are not commensurate with maintaining a positive social position. Other research using the schema-maintenance through compensation model has found that individuals respond to threats to important schemata (e.g., stereotypes) by generating information that at least attempts to compensate for, or balances-out, the threatening information (e.g., Seta et al., 1993, 2003).1 It also may be the case that individuals respond similarly to self-threatening information and generate compensatory self-relevant information. Thus, individuals also may attempt to balance the negative implications of social comparison threats.

According to our view, compensatory effects occur when individuals are both motivated and able to generate (or access) information that has the potential to 
balance-out inconsistent and negative experiences. However, when individuals are not motivated or capable, the inconsistent event can have a direct influence and produces responses that are driven by the inconsistent experience. Although there has been research that supports these assumptions (e.g., Alter \& Seta, 2005; Hughes \& Seta, 2003; Seta et al., 1993; Seta, Seta, \& Goodman, 1998; Seta, Hundt, \& Seta, 1995; Seta \& Seta, 1982, 1993; Seta, Seta, \& McElroy, 2003), research has not tested the role of these processes for the self in a context in which inconsistent experiences were induced by upward social comparisons. The purpose of the following research is to extend this model into the domain of social comparison threat.

Compensation and spreading of activation. It is worthwhile to consider why individuals might be motivated to compensate for upward social comparisons when they have just received objective information that they performed better than average. Why not just accept the objectively positive feedback and ignore the social comparison information that others have performed better than they in the setting? In many cases, this may indeed occur. However, in competitive contexts upward social comparisons can signal an inferior social position that is associated with the loss of social status and the potential for rejection. This is especially likely in a context in which differences between persons' performance levels are salient and consequential. Being outperformed by another can represent an important loss and comparers may attempt to compensate for this loss by providing themselves with an especially positive social position relative to another group member. To successfully accomplish this goal, a suitable target must be available-one that allows performers to access relatively positive self-attributes. Typically, a person holding a relatively inferior social position relative to performers can serve this role. Thus, because an above-average performers' accomplishments are superior in comparison to those of the average person, performers may be able to access self-attributes that are relatively more positive than those of this inferior other.

In addition to motivating performers to compensate for the unfavorable social comparison, however, the negative implications associated with this type of upward comparison can serve as a predictive cue for performers' self-worth by activating negatively valenced self-knowledge information, which spreads outside of awareness and relatively automatically to associatively linked self-concepts. The result of this passive spreading of activation process (e.g., Collins \& Loftus, 1975; Higgins, 1996), is an increase in the availability of negative self-information which, in turn, can constrain attempts to access information that compensates for the unfavorable comparison.

When the negative implications of an unfavorable social comparison are relatively mild, a performer should be capable of accessing information that at least partly balances the unfavorable experience. However, when the negative implications are severe, they may reduce performers' ability to access positive self-attributes and, thus, their self-evaluations may be driven by the negative implications of the unfavorable social comparison resulting in deflated self-evaluations.

Compensation and self-esteem. If a lack of ability to access positive self-attributes constrains compensation following a failure experience, then person variables related to this factor also should affect this capability. Several studies have examined person factors, such as self-esteem, on perceivers' self-evaluations following an experienced failure (e.g., Baumeister, 1982; Brown \& Gallagher, 1992; Brown \& Smart, 1991). In 
general, these studies have found patterns of elevated self-evaluation following an experienced threat for high, but not low, self-esteem persons (for reviews concerning self-esteem, see Hoyle, Kernis, Leary, \& Baldwin, 1999; Kernis, 1995). This may be because low self-esteem persons believe that they have relatively few resources or positive attributes (e.g., Brockner \& Elkind, 1984; Brockner et al., 1998), or because they may not be confident that they can defend their positive self-views-either to themselves or to others (e.g., Baumeister, Tice, \& Hutton, 1989; Brown \& Smart, 1991; Crocker \& Wolfe, 2001). If so, then the inability of low self-esteem individuals to self-enhance would be due to deficiencies in cognitive abilities rather than lack of motivation (e.g., Seta, Donaldson, \& Seta, 1999; Swann \& Schroeder, 1995).2 If deficiencies in cognitive abilities are responsible for the failure of low self-esteem performers to self-enhance, then less "cognitively demanding" conditions may reveal their self-enhancing motivations.

With few exceptions (see Brown \& Gallagher, 1992), the vast majority of studies that have found especially positive self-evaluations following a negative evaluation have compared the responses of persons who have received negative versus positive evaluations (e.g., Baumeister, 1982; Baumeister \& Jones, 1978; Brown \& Smart, 1991; Dunning, Luenberger, \& Sherman., 1995; Tesser, Crepaz, Collins, Cornell, \& Beach, 2000). Although these studies are informative about individuals' selfevaluations following negative versus positive feedback, these studies do not provide information concerning their responses relative to a comparatively neutral control group in a social comparison context. Therefore, in the present studies, we compared the self-evaluations of performers to those who were not exposed to a threatening upward comparison-a control group.

We also provided high and low self-esteem performers with upward social comparisons that represented different levels of negativity and threat, and then measured their self-evaluations relative to an inferior comparison target. A competitive environment was created. In the experimental comparison conditions, relative evaluations were made salient; a relatively high status evaluator was purportedly scheduled to objectively compare differences in the participants' performance relative to those of another person who was always described as performing at a relatively superior level. Therefore, there was competition established between performers and the superior, and there were clear consequences, such as loss of public face, associated with this situation. Thus, it would be difficult for performers to alter the fact that they were different from-and inferior to-the superior. Our expectations were that both low and high self-esteem performers would be motivated to balance this negative experience by positively differentiating themselves from the inferior. However, low self-esteem performersbecause of their limited ability to access positive self-attributes-should be especially likely to be constrained in their ability to compensate. Thus, although they may be capable of compensating when the unfavorable social comparison is relatively mild, they should be less likely to compensate when it becomes more severe. In fact, when exposed to a very unfavorable social comparison, the selfevaluations of low self-esteem persons may be driven primarily by the inferior experience itself. If so, then their self-evaluations would be deflated relative to those of low self-esteem performers who were not exposed to the unfavorable social comparison-a control group. 
Similarity and elevated self-evaluations. Research has shown that upward social comparisons may be interpreted in a positive light leading to positive affect and elevated self-evaluations, such as when individuals focus on the similarities between themselves and the superior person (e.g., Buunk, Collins, Taylor, Van Yperen, \& Dakof, 1990; Buunk \& Ybema, 1997; Collins, 1996; Lockwood \& Kunda, 1997). Thus, performers also may access positive self-attributes and raise their selfevaluations because they believe they are similar to the superior person.

According to the selective accessibility model (e.g., Mussweiler, 2001, 2003; Strack \& Mussweiler, 1997), when perceivers believe they belong in the same category as the standard, they engage in similarity testing and assimilate themselves to the standard; they engage in dissimilarity testing, however, and contrast themselves away from the standard when they perceive that they and the superior are in different categories (e.g., Mussweiler \& Bodenhausen, 2002). In the present studies, we created a competitive situation and placed performers into a different achievement category relative to the superior; they were either one or two ranks below the superior. Thus, we created a situation in which performers would see themselves as distinct from the superior (e.g., Brewer \& Weber, 1994; Brown, Novick, Lord, \& Richards, 1992). Differences between performers and the superior also had external consequences in that performers expected an important evaluator to compare their accomplishments to those of the superior. Thus, this type of setting should not induce performers to see their accomplishments as being interdependent or similar to those of the superior. Rather, they should see their accomplishments as discrepant and inferior to the superior, and they should be concerned about the negative consequences associated with differences in their achievements relative to those of the superior.

Overview of Experiments 1 and 2. Two studies were designed to determine whether performers would elevate their self-views as a compensatory response to an unfavorable social comparison. In addition, we tested the hypothesis that high and low self-esteem participants would differ in their ability to compensate for this comparison threat. Specifically, we expected both low and high self-esteem performers to elevate their self-evaluations when the social comparison threat was relatively mild and expected only high self-esteem participants to be capable of this form of compensation when the threat was relatively severe. In fact, when faced with a relatively severe threat, the self-evaluations of low self-esteem performers may be deflated relative to comparatively neutral control group performers.

In Experiment 2, we measured performers' negative affective reactions as well as their self-evaluations. Comparisons to superior others were expected to produce negative affect. Furthermore, levels of negative affective reactions should be most intense when the difference between the participant and the comparison target is relatively large. Therefore, we expected to observe the most intense negative affective reactions in the condition in which participants were confronted with the accomplishments of a very superior person, less intense negative affect reactions when the comparison target was only somewhat superior, and the least intense reactions when the accomplishments of performers were similar to those of the target. If elevated social comparisons are compensatory in nature, then we should find relationships between experienced negative affect and self-ratings. 


\section{EXPERIMENT 1}

\section{Introduction}

Experiment 1 was designed to determine whether performers would view themselves in a more positive light (relative to the inferior average participant) in response to a threatening upward social comparison than in the absence of such a comparison. It was also designed to determine whether these self-evaluations varied as a function of self-esteem level. In particular, we expected both high and low self-esteem participants to be able to compensate for relatively mild social comparison threats but expected that only our high self-esteem participants would be able to compensate for more severe threats. Thus, we expected Experiment 1 to reveal a self-esteem by social comparison condition interaction.

\section{Method}

Participants and design. One hundred sixteen UNCG female undergraduate psychology students participated in this experiment for partial course credit. Our sample was composed of all women because women composed the vast majority of the available participant pool and we wanted to maintain a homogeneous comparison context. Participants were assigned randomly to experimental conditions. A between-subjects design was utilized in which we varied three levels of social comparison information (no comparison control, superior comparison target, and very superior comparison target). In addition, we divided participants at the median level of self-esteem scores (Median $1 / 432$ ), creating two levels of a self-esteem variable (high and low). In the control condition, participants were given feedback about their performance level but were not given information about another performer. In the social comparison information conditions, participants were given feedback about their performance and that of either a superior or a very superior performer. There were, on average, 8 to 12 participants per session.

Procedure. Individuals participated in small groups of 8 to 12 per session and experimental conditions were randomized within each session. At the beginning of the session, participants were given the Rosenberg (1965) self-esteem inventory. After the completion of this inventory, participants were given a copy of the Social Cognitive Aptitude Test (SCAT). This is a bogus test developed by Crocker and her colleagues (Crocker, Thompson, McGraw, \& Ingerman, 1987). The cover sheet of the SCAT test consisted of basic instructions and portrayed the test as an indicator of social and cognitive abilities. Female students who have been asked about tasks that are diagnostic of cognitive abilities have indicated that they are self-relevant (Seta et al., 1999).

The test consisted of six observations of different couples, and participants were asked to rate the likelihood that each couple would stay together for more than one year. They were then informed that they had 10 minutes to complete the test and told that they would be receiving feedback following the performance phase of the experiment. After completion of this task, test booklets and answer sheets were collected. Participants were told that the tests would be taken to an assistant for grading. After a delay in which participants waited quietly in their seats, results were returned to participants. 
It is important to note that participants were not aware of the other performer's identity, did not observe this performer's activities, and did not interact with the performer in any way. Also, because the experimental manipulations were induced via typed instructions, participants were able to perform the task simultaneously, unaware of how others performed and unaware of the information that anyone else was given. All participants read that the lowest possible score on this test was 50, and the highest possible score was 150 and that the average score of all introductory psychology students at UNCG who took this test was 100. In all conditions, participants read that they received a score of 111 and that the experimenter planned to meet with them to discuss possible reasons for potential differences in performance. In the 111-control condition, they were given their score (111) and the average student's score (100) whereas, in the superior performer conditions, they also were given information that they would be compared with another performer (described as "the other person you will be interacting with"). In the two performance comparison conditions, participants were given information about another performer. In the superior condition, they read that, "the other person you will be interacting with scored 132;" in the very superior condition, they read that, "the other person you will be interacting with scored 143."

Although participants were given the range of possible scores, it still might be difficult for them to objectively evaluate their score relative to that of another performer. Therefore, we provided additional information. In the superior condition, the other person's accomplishments placed her in a category that was one rank above that of participants; whereas in the very superior condition, this information placed her in a category that was two ranks above. In both conditions, participants read that, because of these ranking differences, "the two of you are not in the same performance group."

All participants read that, because they might be asked to perform a conceptually similar task, we wanted them to predict how well they would perform on the subsequent task. They were told that the extent to which they could accurately predict their performance on this task would contribute to their overall performance evaluation. Participants were then presented with two comparative self-evaluation measures. They were asked to evaluate their performance relative to the average other performer by rating their performance relative to the average student who took this test on a 101-point scale (where 0 represented "very below average" and 100 represented "very superior"). They also were asked the likelihood that they would beat the average student who took this test using a similar 101-point scale (where 0 represented "not at all likely" and 100 represented "extremely likely"). 3

\section{Results}

Comparative self-ratings. The two comparative self-rating questions (performance measure and likelihood measure) were entered into a MANOVA including the between-participant factors of self-esteem (top $1 / 2$ and bottom $1 / 2$ ) and three levels of performance comparison: no other person; superior (132); and very superior performer (143). Consistent with expectations, this analysis revealed a significant Self-Esteem x Performance Comparison multivariate interaction $F(2,110)=4.24$, p5.02. No other effects reached significance with alpha set at .05; there was, however, a performer comparison condition main effect that approached significance, $F(2,110)=2.90, p<.06$. The individual univariate tests were examined and 
revealed significant Self-Esteem6Condition interactions for both the relative performance measure, $F(2,110)=3.27, p<.05$, and the likelihood of outperforming the average student measure, $F(2,110)=3.64, p<.05$. No other effects were significant in the univariate analyses.

Following the suggestions of Keppel (1991), the significant interactions were decomposed into a set of logical interaction comparisons, similar to simple effects tests. Specifically, "miniature" factorial designs were created from the original complex factorial structure in order to isolate the features of the independent variables responsible for the 2-way interactions.

First, relative performance and likelihood ratings were analyzed as a function of self-esteem level (high and low) within only the control and superior conditions. These analyses revealed a significant comparison condition main effect for the likelihood measure, $F(1,73)=4.76, p<.05$, and no significant interactions involving level of self-esteem for either measure. As may be seen in Table 1, participants raised their relative self-evaluations from the control to the superior condition, but this effect reached significance only for the likelihood measure.

TABLE 1 Mean Ratings of Relative Performance and Out-performance Likelihood as a Function of Self-esteem and Performance Comparison Feedback

\begin{tabular}{llc}
\hline & \multicolumn{2}{c}{ Self-esteem Level } \\
\cline { 2 - 3 } & High & Low \\
\hline Out-performance Likelihood Measure & & \\
No performance comparison & 51.2 & 56.9 \\
Superior & 62.7 & 66.6 \\
Very superior & 67.2 & 49.7 \\
Relative Performance Measure & & \\
No performance & 58.6 & 61.9 \\
Superior & 58.6 & 65.7 \\
Very superior & 65.6 & 54.7 \\
\hline
\end{tabular}

In contrast, the corresponding interaction contrast comparing the control and very superior conditions produced a significant interaction between Self-Esteem Level and Comparison Condition for the likelihood measure, $F(1,74) \frac{1}{4} 46.01, p 5.05$, and approached significance for the relative performance measure, $F(1,74) \frac{1 / 4}{3} 3.86$, p $1 / 4.055$. Follow-up simple comparisons revealed that high self-esteem participants significantly raised their self-evaluations on the likelihood of outperforming the average student measure from the control to the very superior condition, $F(1,74) \frac{1}{4} 12.77$, p 5.05 , whereas low self-esteem participants descriptively decreased their estimates of out-performing the average student from the control to the very superior condition, $F(1,74) 1 / 41.95, p 5.20$.

A final orthogonal interaction contrast was performed analyzing high and low selfesteem 
participants' self-evaluations as a function of the superior (132) and very superior (143) comparison conditions. This analysis revealed significant interactions between Condition and Self-Esteem for both the relative performance measure, $F(1,73)=5.95, p<.02$, and for likelihood estimates of out-performing the average other (i.e., likelihood measure), $F(1,73)=4.67, p<.05$. Follow-up simple comparisons showed that low self-esteem participants significantly lowered their selfevaluations from the superior to very superior condition in terms of their likelihood estimates of out-performing the average student, $F(1,73)=6.55, p<.05$, and on the relative performance measure, $F(1,73)=4.85, p<.05$. In contrast, high self-esteem participants' evaluations were not lower in the very superior versus the superior condition. There was no difference between these conditions on the likelihood measure $\mathrm{F}<1$ whereas on the relative performance measure, participants raised their self-evaluations from the superior to the very superior conditions, $F(1,73)=3.62, p<.07$.

We also treated self-esteem as a continuous variable in a multiple regression analysis in which we dummy-coded the comparison condition and included the interaction between self-esteem and comparison conditions as predictor variables. The average of the two self-evaluation measures was used as the dependent variable. (The correlation between the two measures was $r=.63, p<.01$.) This analysis revealed a significant Self-Esteem6Condition interaction, $F(2,113)=16.91$, $p<.01$. Thus, the regression approach and the ANOVA analyses produced conceptually and statistically similar outcomes.

In sum, these results suggest that both high and low self-esteem participants responded similarly to comparison information that another person performed one rank above them. However, when the social comparison feedback indicated that another person was very superior (i.e., two ranks above the participants), high and low self-esteem participants showed different patterns of self-evaluations. Relative to controls, low self-esteem participants tended to lower their ratings of self (both relative performance and likelihood measures) whereas their high self-esteem counterparts significantly raised their estimates of out-performing the average student and tended to raise their relative performance evaluation.

\section{Discussion}

In this experiment we sought to determine whether elevated self-evaluations would follow from unfavorable social comparisons. To answer this question, we included a control group in which participants were not exposed to a social comparison threat as well as conditions in which the level of social comparison threat was varied. Results indicated that both high and low self-esteem participants had especially positive self-evaluations following mild social comparison threats. They did not, however, have similar self-ratings following exposure to a very superior (143) performer. In the more extreme upward comparison context, high self-esteem performers had elevated self-ratings whereas their low self-esteem counterparts did not. In fact, low self-esteem performers tended to demonstrate deflated self-ratings after exposure to a very superior performer. Therefore, when the failure experience itself was not especially intense (as in the superior performer condition), high and low self-esteem persons did not differ in their tendencies to compensate. In contrast, when the discrepancy between individuals' performance and that of the performer 
was very large, as in the very superior condition, only high self-esteem persons were able to elevate their self-evaluations.

According to our analysis, these results were obtained because high self-esteem persons had the ability to access positive self-attributes whereas low self-esteem persons did not. And, because low self-esteem persons were constrained in their ability to access positive self-attributes, the failure experience had a direct influence, resulting in deflated self-views.

The procedures used in Experiment 1 make it unrealistic to believe that our participants learned specific skills from observing the superior performer (e.g., Bandura, Blanchard, \& Ritter, 1969). Participants were only given information about the superior performers' scores, not about the superior performers' activities. And, because all of our participants were given the full range of potential scores on this task, it is unlikely that there were differences in participants' knowledge concerning scores that could or could not be attained as a function of comparison conditions. In addition, by placing participants and the superior performer into different categories and by making differences that existed between them impactful, we made it likely that their elevated self-ratings were the result of the negative implications associated with their inferior position relative to the superior.

\section{EXPERIMENT 2}

\section{Introduction}

Experiment 1 did not provide direct evidence for the hypothesis that participants' elevated self-ratings were driven by the negative psychological implications of unfavorable social comparisons (i.e., negative affect). Therefore, Experiment 2 was designed to determine whether elevated self-ratings were related to the negative affect generated by the social comparison threats. This finding would support the reasoning that heightened self-evaluation effects occurred as a form of compensation for the negative implications of unfavorable social comparisons. In addition, this finding should rule out explanations based upon views that participants believed that they were similar to the superior performers (e.g., Buunk et al., 1990; Collins, 1996); this type of assimilation affect should not produce negative affect. Because we explicitly made salient differences in the rank of the participants and the comparison targets, we did not expect results that would support assimilation effects. Thus, we expected the most intense negative affective reactions when participants were to be compared to a very superior person, a less intense reaction when the comparison target was only somewhat superior and the least intense reaction when the scores of performers were described as similar to those of the comparison target.

In Experiment 1, superior and very superior condition participants were given two sources of comparison information (the score of the average person and the score of the comparison target) whereas participants in the no performance comparison condition were given only one (the score of the average person). Furthermore, although participants in the superior and very superior performer condition did not interact with the performer, they expected this interaction in the future. Participants in the no performance comparison condition were not given this information. Therefore, across conditions, participants in Experiment 1 may have had different 
expectations concerning their future interactions. To be sure that this was not a critical factor, Experiment 2 was designed to give all of our participants identical information concerning their future interactions; all participants were told that they would meet with the experimental assistant and were not told that they would interact with the comparison target in the future. And to keep the presence (or absence) of the performer constant across conditions, we changed the "no performance comparison" condition to one in which participants learned about the performance of another individual. In this condition, the score given to participants was relatively similar to the one given to the performers. Consequently, participants should demonstrate a lesser degree of negativity in this situation, relative to situations in which they were clearly out-performed (superior and very superior condition).

\section{Method}

Participants and design. One hundred forty-three female undergraduate students participated in this experiment for partial course credit. We varied three levels of performance comparison (109: similar, 132: moderately superior and 143: very superior) in a between-subjects design. In addition, we measured participants' self-esteem and included it as a variable in the design, resulting in a 3 (comparison condition: similar, moderately superior, and very superior) 62 (self-esteem: high or low, based on a median split at 32) between-subjects design.

Procedure. The procedure of Experiment 2 was similar to that of Experiment 1 and participants were assigned randomly to experimental conditions. Following the administration of Rosenberg's self-esteem inventory, participants took the self-relevant test (SCAT) and were provided (bogus) feedback about the range of scores possible on the test $(50-150)$. They were provided with the average student's score (100). Across all conditions, participants were told that they had scored 111, and were given the score of another person. In the similar performance comparison condition, participants were told that the other participant had scored 109; in the superior condition they were told that the other person had scored 132; in the very superior condition they were told that the other person had scored 143. Following the presentation of these results, participants in the similar condition were informed that they were in the same performance category as the other performer whereas performers in the superior and very superior comparison conditions were told that they were not in the same performance category. As in Experiment 1, we further highlighted differences in the scores of the superior and very superior performers; superior condition participants were told that they were in a performance category that was one rank below the other performer whereas those in the very superior conditions (143) were informed that they were two ranks below the other performer. Therefore, not only was the other performer's score higher in the very superior versus superior condition, but the other performer was described as having a superior rank as well.

All participants were told that the experimenter would meet with them to discuss possible differences between their performance and that of the other person. Following this information, participants were told that they might perform this task again and that part of their overall score would be determined by their ability to predict their own performance on a different, but conceptually similar, test; the 
second test would contain questions that were different from those on the first test but measured the same performance abilities as the first test. Following the collection of the dependent variables described below, participants were thanked and fully debriefed.

Measures. As a manipulation check, participants were asked how they would rate the score the other person obtained on the first test, relative to their own score, where 0 represented "very below average," 50 represented "about equal to," and 100 represented "very superior." 4 To determine their affective state, participants were asked to consider their present feelings in light of their performance and that of the other person and to rate each of four adjectives in terms of how characteristic each was of their present feelings. The ratings were made on a 7-point scale where 1 represented "not at all characteristic" and 7 was "totally characteristic." We included two negatively valenced adjectives ("frustrated" and "not at all pleased"as a qualified adjective) and two positively valenced ones as well ("happy" and "satisfied").

We then asked participants several self-rating questions. First, they were asked two comparative questions worded in the manner described in Experiment 1. They were also asked two additional questions: how they would rate their performance and how they would rate the performance of the average student who took this. Participants' responded on a 101-point scale where 0 represented "very below average" and 100 represented "excellent."

The evaluative test used in this research involved both cognitive and social competencies. Therefore, we also included questions that involved social competencies. Questions of this type have been used in prior research dealing with evaluations of self and others (Alicke, Klutz, Breitenbecher, \& Yurak, 1995). Participants were asked to rate themselves relative to the average person on the attributes of "dependability" and "maturity." Specifically, they were asked how they would rate themselves as a dependable person, compared to the average student, and how they would rate their maturity compared to the average person on a 101-point scale where 0 represented "not at all" and 100 represented the extreme ("extremely" for the dependability question or "or exceptionally high" for the maturity question). They were also asked questions about dependability and maturity in a noncomparative manner ("How dependable (mature) would you rate yourself? How dependable (mature) would you rate the average student?"). The 101-point scale described above was adapted to these questions.

\section{Results and Discussion}

Manipulation checks. We performed a 2 (high and low self-esteem) $\times 3$ (similar, superior, and very superior performance comparison condition) ANOVA in which we used participants' comparative ratings of how they judged the performance of the other person relative to their own performance as the dependent variable. The analysis revealed a performance comparison main effect, $F(2,105)=16.7, p<.001$. There were no other significant effects (all ps greater than .5). Participants in the similar performance comparison condition rated the other person's performance as "about equal" to their own $(\mathrm{M}=54.84)$ and indicated a lower relative standing in comparison to ratings of the performer described in the superior $(M=64.72)$ and 
very superior $(M=78.13)$ conditions, $F(1,105)=12.1, p<.05 ; F(1,105)=64.3$, $p<.001$, respectively. In addition, participants in the very superior performance comparison condition rated the other performer as having a higher relative ranking relative to those in the superior condition, $F(1,105)=22.2, p<.001$.

Thus, the manipulations were impactful and established differences in participants' perceptions about the comparison other's performance levels relative to their own performance. Furthermore, these data demonstrate that participants did not put themselves in the same category as the superior performers described in the superior and very superior conditions. These data are important in ruling out explanations based upon participants' assimilation to superior others in these conditions. If this had been the case, one would expect that participants in the very superior and superior conditions would have rated themselves as being approximately equal to their comparison target. However, this was not the case. As we will discuss, performers' negative affect scores also do not support this conclusion. Self-evaluations. A primary question addressed in this study concerned whether perceivers' self-evaluations following unfavorable social comparisons would be elevated as a function of the level of discrepancy between the comparer and the comparison target. In addition, we examined the role that participants' level of selfesteem played in determining the effects of an unfavorable social comparison. To address these questions, we examined participants' self-evaluations relative to the "average person." Questions that tapped these dimensions asked for separate ratings of self and the average student and were used to obtain a difference score, calculated by subtracting participants' ratings of the average student from their judgments of themselves for each specific evaluation dimension (for performance, for maturity, for dependability). The difference score measures and the comparative rating measures were significantly related, alpha $1 / 4.65, \mathrm{p} 5.05$, and were, therefore, averaged to create a single composite index of relative self-evaluation.

These composite scores were entered into a between-subjects ANOVA including three levels of performance comparison information (control-similar, superior-132, very superior-143) and two levels of self-esteem (high and low). The analysis revealed a significant main effect of level of self-esteem, $F(1,137)=10.58, p<.01$; high selfesteem participants rated themselves more positively $(\mathrm{M}=48.98)$ than did their low self-esteem counterparts ( $M=44.38)$. In addition, the main effect of performance comparison condition was significant, $F(2,137)=5.05$, p 5.01 . Tukey HSD tests revealed significant differences between evaluations in the control-similar condition ( $\mathrm{M} 1 / 444.88)$ and the superior-132 condition (49.80), p5.05, but did not reveal significant differences in comparisons with the very superior-143 condition $(\mathrm{M}=45.35)$. Therefore, participants raised their self-evaluations following upward comparisons to a superior performer, but not following comparisons with a very superior performer.

These main effects were qualified by a significant Self-Esteem6Performance Comparison Condition interaction, $F(2,137)=6.09, p<.01$. Because this interaction was expected from Experiment 1 and from our theoretical perspective, we conducted planned and orthogonal contrasts on specific cell means. As may be seen in Table 2, although there were no significant differences between the self-evaluations of high and low self-esteem participants in the control condition $(F<1)$, both high and low self-esteem participants' self-evaluations were significantly more positive in the superior than in the control condition, $\mathrm{Fs}(1,137)=4.07,4.15$, respectively for 
high and low self-esteem participants ps5.05. Comparisons also indicated low selfesteem participants' self-evaluations decreased significantly from the superior to the very superior comparison condition, $F(1,137)=14.85, p<.05$ and were lower than those obtained in the control condition, $F(1,137)=3.98, p<.05$. In contrast, for high self-esteem participants, self-evaluations were significantly more positive in the very superior than in the control condition, $F(1,137)=5.9, p<.05$. These data provide a conceptual replication of the results of Experiment 1 and support our theoretical analysis.

We also performed a regression analysis on the composite self-evaluation scores treating self-esteem as a continuous variable and including comparison condition (dummy-coded) and the Self-esteem6Comparison Condition interaction term as predictor variables. Consistent with the results of the ANOVA, we obtained a significant Self-esteem6Comparison Condition effect, $F(2,137)=8.17, p<.01 .^{5}$

Negative affect. To obtain a negative affect score for each participant, we reverse scored the two positively valenced adjectives. Because there was a significant positive correlation between the reverse scored positively valenced and negatively valenced adjectives, $r=.67, p<.01$, we combined these adjectives in computing the negativity score for each participant. We performed a 2 (high and low self-esteem)63 (similar, superior and very superior performance comparison) between-subjects ANOVA with participants' negative affect scores as the dependent variable. The analysis revealed a performance comparison main effect, $F(2,137)=20.41, p<.01$. No other effects were significant.

TABLE 2 Average Comparative and Absolute Difference Ratings as Function of Performance Comparison Condition and Self-esteem Level

\begin{tabular}{lcc}
\hline & \multicolumn{2}{c}{ Self-esteem } \\
\cline { 2 - 3 } Comparison Condition & High & Low \\
\hline Similar & 45.31 & 44.46 \\
Superior & 50.45 & 49.15 \\
Very superior & 51.20 & 39.56 \\
\hline
\end{tabular}

If our procedure was effective then performers should be concerned about the negative consequences associated with differences between their accomplishments and those of the superior. Thus, they should experience heightened levels of negative affect when they anticipate a comparison to this person; and their affective reactions should be most intense when there are large differences between their accomplishments and those of the superior.

Tukey HSD tests revealed that our procedure was effective. The comparison main effect was due to the fact that participants experienced a lesser degree of negative affect in the similar $(\mathrm{M}=9.6)$ than in the superior $(\mathrm{M}=13.1)$ and very superior performance comparison conditions $(M=15.5)$, ps <.05. In addition, participants reported higher levels of negative affect in the very superior than in the superior performance comparison condition, p5.05. 
It is important to note that, although differences in the reactions of high and low self-esteem individuals to failure are relatively common (e.g., Kernis \& Waschull, 1995), participants' level of self-esteem did not affect their negative affective reactions in the present study. Our procedure created a situation that was competitive with clear and important external consequences. In this situation, the threat to performers' social standing was strong enough to eliminate negative affective differences related to self-esteem. We would note, however, that different patterns of negative affect scores could be obtained under different conditions. It is also important to note that although the omnibus ANOVA revealed no differences in high and low self-esteem participants' level of negative affect $(F<1)$, the analyses of self-evaluations revealed an interactive effect of self-esteem. This pattern suggests that the negative implications of the unfavorable social comparison motivated both low and high self-esteem performers to compensate for the unfavorable social comparison. Because of differences in their abilities to access positive self-attributes, however, high self-esteem performers were able to compensate whereas low self-esteem performers were not. Thus, differences between low and high self-esteem performers' self-evaluations occurred because of differences in their abilities to compensate for negative affect, not because they differed in the amount of experienced negativity. 6 Furthermore, our procedure provided performers with feedback about the accomplishments that were highly believable, as evidence by the fact that low and high self-esteem performers had similar evaluations in the control conditions of Experiments 1 and 2.

Correlation analysis. If increases in self-evaluations result from motivations to compensate for the negative affect generated by unfavorable social comparisons, then we should find positive correlations between self-evaluations and felt negative affect. In support of this reasoning, in the superior performer comparison condition, there were positive correlations between the negative affect and comparative evaluation scores of both low, $r=.40, p<.05$; and high self-esteem participants, $r=.48, p 5.05$. These patterns suggest that as negative affect increased, so too did the positivity of participants' comparative evaluations. Therefore, both high and low self-esteem participants were able to engage in compensatory self-evaluations when the threat posed by the social comparison was relatively mild. This relationship is in line with our predictions.

We also expected a divergence in the patterns of high and low self-esteem participants' self-evaluations under conditions of more extreme social standing threat (very superior condition). In this case, we predicted and found low self-esteem persons' self-evaluations to be relatively low (in relation to the superior condition and in relation to high self-esteem participants).

In support of this reasoning, the comparative evaluations of low self-esteem participants decreased as their experienced negative affect increased, $r=7.48$, p5.05. In contrast, the self-evaluations of high self-esteem participants were positively, although not significantly, correlated with negative affect, $r=+.13$. The divergence in the correlations obtained for high and low self-esteem performers supports the view that low self-esteem performers were less capable of accessing positive self-attributes than their high self-esteem counterparts. The fact that the positive correlation obtained for high self-esteem performers was not significant suggests that the highly unfavorable social comparison may have constrained (or 
started to constrain) the capabilities of some of our high self-esteem performers as well. 7

\section{Summary Results}

The combined results of this experiment revealed several significant effects. First, because of their inferior social position vis-a` -vis the superior low and high self-esteem participants had heightened and similar levels of negative affect. Further, as our manipulation check measures indicated, they also had depressed ratings of themselves relative to the superior. In response to a modestly unfavorable comparison, both low and high self-esteem participants raised their ratings relative to the inferior average person. However, an especially unfavorable social comparison induced elevated ratings for high self-esteem participants, but not for their low self-esteem counterparts. In fact, low self-esteem participants had deflated ratings in this condition. Furthermore, our results supported the conclusion that differences in ability, not perception or motivation, were the primary determinants of differences in the compensatory responses of high and low self-esteem individuals.

\section{GENERAL DISCUSSION}

In Experiments 1 and 2, we expected performers to be concerned about the negative consequences associated with the difference between their accomplishments and those of a superior other. To achieve this end, we created a competitive situation that was overseen by an evaluative other. The results of Experiment 2 supported the effectiveness of our manipulation in that performers' negative affective reactions were most intense in the condition in which they were confronted with the accomplishments of a very superior person who was two ranks above them, less intense when the comparison target was one rank above, and least intense when the accomplishments of performers were similar to those of the comparison target. The results provide evidence that our performers considered their accomplishments to be different from those of the superior and that these differences had negative implications to them.

Experiments 1 and 2 were also designed to determine whether both high and low self-esteem persons were capable of elevating their self-evaluations vis-a` -vis an inferior person when the social comparison threat was relatively mild. In this situation, the failure experience was not especially intense in that performers' accomplishments were only one rank below those of the superior comparison target. Furthermore, the intensity of the unfavorable social comparison was softened by the fact that participants' absolute performance score was described as somewhat better than average. Therefore, although the feedback concerning the task was impactful to participants and was significantly more negative than in the control condition, it was not very intense. And because it was not especially intense, low self-esteem performers were able to elevate themselves relative to an inferior, leading them to demonstrate a compensatory response that was comparable to their high self-esteem counterparts. In this situation, support for compensation was obtained because the self-evaluations of participants who experienced an unfavorable social comparison was more positive than those who had not experienced this threat. Further support for compensation was obtained by considering the relationship between participants' 
self-evaluations and their affective reaction to the mildly unfavorable social comparison. As their negative affective reaction to the unfavorable social comparison increased, their positive self-evaluations vis-a` -vis an inferior increased.

Prior work (e.g., Seta et al., 1999) has shown that self-enhancement tendencies are influenced by how directly tied the information is to performers' self-views (see Crocker \& Wolfe, 2001, for a discussion of how contingencies of self-worth influence perceivers' reactions). The present results suggest that, when a task has important consequences vis-a '-vis performers' social standings, low self-esteem performers were capable of compensating for unfavorable upward comparisons as long as its intensity was not too great and a "compensatory friendly target," such as an inferior other, was available. However, only high self-esteem persons were capable of compensating when there was a large discrepancy between their achievement level and that of the comparison target, as in the very superior performance comparison condition. This was the case even though both high and low self-esteem participants experienced equivalent levels of negative affect in the superior comparison condition.

The schema maintenance through compensation model. In addition to building on prior work concerning compensatory reactions that implicate the self (e.g., Baumeister, 1982; Baumeister \& Jones, 1978; Brown \& Smart, 1991; Seta et al., 1999), the results of the present studies add to research following from a schemamaintenance through compensation analysis (e.g., Alter \& Seta, 2005; Hughes \& Seta, 2003; Seta \& Seta, 1992, 1993; Seta et al., 1998, 2003). According to this approach, three events are necessary for compensation to occur (Seta \& Seta, 1992, 1993; Seta et al., 1993, 2003): (1) an event or experience must be perceived to be inconsistent with a schema, goal, or expectation; (2) the person must have an implicit or explicit motivation to maintain the schema; and (3) the person must have the ability to generate information that can partly balance the inconsistent event. All three of these conditions are met by the conceptual variables explored in this research and the differences in the abilities of high and low self-esteem persons to compensate for failure experiences fit squarely within the domain of this model. Thus, a parsimonious account of compensatory motivations in the context of unfavorable social comparisons is offered by the schema-maintenance through compensation model (Seta \& Seta, 1992, 1993; Seta et al., 1993, 2003).

The results of several studies have supported the motivational assumptions of this model (e.g., Seta \& Seta, 1982, 1993; Seta et al., 1993, 2003). For example, when confronted with information that was inconsistent with their stereotype, individuals compensated for an inconsistent event (via expectations and attributions) when they were motivated to maintain the stereotype, but not when they were not motivated (e.g., Seta et al., 1998, 2003). Other research (Seta \& Seta, 1982) has demonstrated that failure can induce compensation in a context in which children worked for a very positive goal (a valuable toy); children inflated the goal's worth after experiencing either a relatively mild or relatively severe failure experience. Because of their motivation to maintain consistency between input (cost) and obtained output (the toy), participants compensated for the failure experience by raising the perceived worth of the goal. Studies also have supported the constraint assumptions of this analysis. A person's ability to generate compensatory information can be constrained by the predictive cue properties of the inconsistent act. For example, an inconsistent act for which a particular group member is directly responsible is especially predictive of this group member's character and future actions; however, it is only marginally 
predictive of the characters of fellow group members-those who are only indirectly related to the inconsistent act(s) via their group affiliation. Thus, perceivers have been shown to expect compensation from a fellow group member, but not from the group member who was associated directly with the inconsistent action (e.g., Seta \& Seta, 1993; Seta et al., 2003).

Compensation also has been shown to be constrained by the properties of compensatory targets. Because there are more aspects to discover about highly valued goals, it took larger amounts of effort (input) to constrain individuals from matching the perceived worth of the goal to their efforts when the goal was high versus low in value (e.g., Seta \& Seta, 1982; Seta et al., 1993). When compensation is constrained, perceivers' evaluations have been shown to be driven by the inconsistent experience, itself. This has been shown to result in a goal's devaluation following either large expenditures of effort or failure experiences (Seta \& Seta, 1982; Seta et al., 1993), in lowered expectations about the future behavior of a group member who was directly responsible for an inconsistent action (e.g., Seta \& Seta, 1993; Seta et al., 1998) and in less positive internal attributions concerning the behavior of an individual who was directly associated with the negative and inconsistent behaviors of multiple group members (Seta et al., 2003).

The above-described results, coupled with those of the present studies, suggest that compensation is a motivated process that occurs whenever inconsistency threatens either social or self-schemata; just as perceivers in past studies were motivated to expect compensation after they were given information that was inconsistent with their stereotype, performers in the present studies were motivated to compensate following an unfavorable social comparison. And, just as perceivers have been shown to be constrained in the ability to compensate for highly inconsistent behaviors of a member of a stereotyped group, low self-esteem performers were constrained in their ability to compensate following a relatively severe social comparison threat. Participants' responses in both the present research and in our research dealing with violations of stereotypes demonstrated the direct implications of inconsistent experiences as well as the direct implications of the failure experience on participants' judgments.

In the present research, as in our past work, we see two processes at work. One process that attempts to compensate for the schema-threatening event and one that is driven directly by the nature of the inconsistent or threatening event per se. For example, following a failure experience, the process of compensating for the negative experience is constrained by the process of the failure experience spreading automatically to associatively linked knowledge units. Thus, to understand the effects of a failure experience, we must understand the interplay between these two processes. In the present studies, the intensity of the unfavorable social comparison (a contextual factor) coupled with self-esteem (a person factor) influenced the relative strengths of these two processes.

Better-than-average effects. The better-than-average (BTA) effect refers to the finding that, across a variety of dimensions, people believe, on average, that they have characteristics that are more positive than those of others (e.g., Alicke, 1985; Alicke et al., 1995; Allison, Messick, \& Goethals, 1989; Codol, 1975; Gibbons, Helweg-Larsen, \& Gerrard, 1995; Goethals, Messick, \& Allison, 1991; Messick, Bloom, Boldizar, \& Samuelson, 1985; Weinstein, 1980). It is a robust phenomenon 
that has been observed across a variety of domains, including estimates of the likelihood of positive and negative events (Perloff \& Fetzer, 1986), and intelligence and fairness (Allison et al., 1989).

Cognitive and motivational reasons have been implicated as the cause of this general effect (Klar \& Giladi, 1997, 1999; Wills, 1981). To the extent that the BTA effect is a motivated bias, it should be influenced by threats to participants' relative social standing. Consistent with this reasoning, we found that, compared to a control group, both low and high self-esteem performers elevated their self-worth relative to the average performer following a moderately threatening upward comparison.

These effects occurred under conditions in which the participants received performance feedback that was objectively positive and better than the average. In raising their self-evaluations, they indicated that they were "better than better-than-average." However, because low self-esteem persons were constrained in their ability to compensate, only high self-esteem persons elevated their self-worth following a relatively severe threat. Therefore, self-esteem and the magnitude of the threatening experience influenced perceivers' "better than average" perceptions.

\section{Summary}

Social comparison information is readily available in our everyday environment. And it is often the case that the persons with whom we interact possess attributes and abilities that are superior to our own. This is especially true for persons in very competitive environments. The results of the present studies suggest that, when faced with an inferior social position, the intensity of the unfavorable social comparison coupled with self-esteem determined whether performers would or would not compensate for their unfavorable position, by inflating their self-ratings relative to an inferior. Certainly, the idea that individuals seek to maintain positive and consistent characteristics of themselves and the social world is not unique. This view has a long tradition in social psychology (e.g., Abelson, Aronson, McGuire, Newcomb, Rosenberg, \& Tannenbaum, 1968; Aronson, 1969; Festinger, 1957). Building on this tradition, the present research allows us to more fully understand when individuals will and will not be able to compensate when faced with threats of a social nature. 


\section{NOTES}

1. Individuals also are motivated to avoid events that are inconsistent with important schemata.

2. Seta et al. (1999) found that low self-esteem persons demonstrated a stronger selfenhancement response relative to high self-esteem persons on a task that was low in self-relevance but only high self-esteem persons were able to self-enhance in a highly self-relevant task. The low self-relevance task used in the Seta et al. (1999) study was conceptually similar to the mildly dissonance producing situation employed in the Steele, Spencer, \& Lynch (1993) study, in which low self-esteem persons were seen to be especially inclined to rationalize the negative implications of a decision.

3. In the superior and very superior conditions, we also asked participants questions about their performance in relation to the superior performer. Importantly, their expectation of the score they would receive was not correlated with their comparative self-evaluation suggesting that comparative evaluations were not the result of an association with the superior performer. We have not discussed these measures in detail because, in Experiment 2, we provided a more direct test of whether performers' elevated self-ratings were a response to an upward comparison threat or a result of an association with a superior performer.

4. Several participants appeared to misunderstand our manipulation check questions. Inspection of the original question for the first 32 participants indicated that a few participants did not respond. Therefore, we revised our question to make it more understandable for participants and substituted this wording for the remainder of the participants. Clarity was achieved by rewording the question and providing a midpoint on the scale. We based our manipulation check analysis on the revised question.

5. For exploratory purposes, we also performed an ANOVA considering the task related (e.g., performance ratings) versus indirectly related (dependability, maturity) attributes as repeated measures as an additional component of the design. Although there was a marginally significant main effect of the measure variable $F(1,137)^{1 / 43.06}$, p5.10, there were no significant interactions involving either self-esteem or performance condition and the measure variable. This dimension may not have mattered in the present study because the attributes of dependability and maturity do have some implications for task performance, given the SCAT involved making judgments about the likelihood of couples remaining in relationships and was described as measuring both social and cognitive skills. Thus, these measures involve the same construct. In addition, these questions were answered in close temporal proximity and were not presented as distinctly different from one another. The major findings involving the significant interaction between self-esteem and condition, of course, still held in this analysis and provided support for the major hypothesis underlying this research.

6. Our affect measure involved participants' ratings of how characteristic each of four adjectives (two negatively valenced and two positively valenced ones) was of their present feelings. The measure was effective in that large differences were obtained across conditions. However, we asked participants to consider their present feelings in light of their performance and that of the other person. A question arises as to whether similar findings would be obtained if we did not give them this directive. In an earlier 
pilot study participants were asked to rate adjectives in terms of how characteristic they were of their present feelings without being asked to consider their feelings in light of their performance vis-a` -vis the other performer. This study included a neutral control condition and one in which participants were compared to a mildly superior performer. As in the present study participants reported higher levels of negative affect and high self-evaluations relative to an inferior in the mildly superior condition than in the control condition. Rating how characteristic adjectives are to participants' feelings is a common measure of affect. However, it can be less sensitive than physiological measures in that participants may attempt to conceal their true feelings and behave in a socially appropriate manner. Thus, even though this measure was sensitive in the present study, a more subtle measure may be needed in situations in which participants' are attempting to mask their affective reactions.

7. Although not the primary question motivating this research, the design of Experiment 2 allowed us to assess how the target of rating (self and average other) was affected by social comparison information. Specifically, individuals can compensate for unfavorable social comparisons by elevating/accessing positive aspects about themselves and/ or by creating/accessing negative aspects of others. The general findings from the analysis of self - other ratings indicated that only high self-esteem persons were able to elevate their self-ratings following an unfavorable social comparison

\section{REFERENCES}

Abelson, R. P., Aronson, E., McGuire, W. J., Newcomb, T. M., Rosenberg, M. J., \& Tannebaum, P. H., (Eds.). (1968). Theories of cognitive consistency: A source book. Chicago, IL: Rand McNally.

Alicke, M. D. (1985). Global self-evaluation as determined by the desirability and controllability of trait adjectives. Journal of Personality and Social Psychology, 49, $1621-1630$.

Alicke, M. D., Klutz, M. L., Breitenbecher, D. L., \& Yurak, T. J. (1995). Personal contact, individuation, and the better-than-average effect. Journal of Personality and Social Psychology, 68, $804-825$.

Allison, S. T.,Messick, D. M., \& Goethals, G. R. (1989). On being better but not smarter than others: The Muhammad Ali effect. Social Cognition, 7, 275 - 295.

Alter, R. J., \& Seta, C. E. (2005). Compensation for inconsistencies: The effects of stereotype strength on expectations of applicants' job success and satisfaction. Sex Roles, 53, 79 - 88.

Aronson, E. (1969). The theory of cognitive dissonance: A current perspective. In L. Berkowitz (Ed.), Advances in experimental social psychology (Vol. 4, pp. 1 - 34). New York, NY: Academic Press.

Bandura, A., Blanchard, E. B., \& Ritter, B. (1969). Relative efficacy of desensitization and modeling approaches for inducing behavioral, affective, and attitudinal changes. Journal of Personality and Social Psychology, 13, 173 - 199. 
Baumeister, R. F. (1982). Self-esteem, self-presentation, and future interaction: A dilemma of reputation. Journal of Personality, 50, $29-45$.

Baumeister, R. F., \& Jones, E. E. (1978). When self-presentation is constrained by a target's knowledge: Consistency and compensation. Journal of Personality and Social Psychology, $36,608-618$.

Baumeister, R. F., Tice, D. M., \& Hutton, D. G. (1989). Self-presentational motivations and personality differences in self-esteem. Journal of Personality, 57, $547-579$.

Brewer, M. B., \& Weber, J. G. (1994). Self-evaluation effects of interpersonal versus intergroup social comparison. Journal of Personality and Social Psychology, 66, 268 - 275.

Brockner, J., \& Elkind, M. (1984). Self-esteem and reactance: Further evidence of attitudinal and motivational consequences. Journal of Experimental Social Psychology, 21, 346 - 361.

Brockner, J., Heuer, L., Siegel, P. A., Wisenfeld, B., Martin, C., Grover, S. et al. (1998). The moderating effect of self-esteem in reaction to voice: Converging evidence from five studies. Journal of Personality and Social Psychology, 75, 394 - 407.

Brown, J. D., \& Gallagher, F. M. (1992). Coming to terms with failure: Private selfenhancement and public self-effacement. Journal of Experimental Social Psychology, 28, $3-22$.

Brown, J. D., Novick, N. J., Lord, K. A., \& Richards, J. M. (1992). When Gulliver travels: Social context, psychological closeness, and self-appraisals. Journal of Personality and Social Psychology, 62, $717-727$.

Brown, J. D., \& Smart, S. A. (1991). The self and social contact: Linking self-representations to prosocial behavior. Journal of Personality and Social Psychology, 60, $368-375$.

Buunk, B. P., Collins, R., Taylor, S. E., Van Yperen, N. W., \& Dakof, G. A. (1990). The affective consequences of social comparisons: Either direction has it ups and downs. Journal of Personality and Social Psychology, 59, 1238 - 1249.

Buunk, B. P., \& Ybema, J. F. (1997). Social comparison and occupational stress: The identification - contrast model. In F. X. Gibbons \& B. P. Buunk (Eds.), Health, coping, and well-being: Perspectives from social comparison theory (pp. 359 - 388). Mahwah, NJ: Lawrence Erlbaum Associates, Inc.

Codol, J. (1975). On the so called "superior conformity" of the self behavior: Twenty experimental investigations. European Journal of Social Psychology, 5, 457 - 501.

Collins, R. L. (1996). For better or worse: The impact of upward social comparison on selfevaluations. Psychological Bulletin, 119, $51-69$.

Collins, R. L., \& Loftus, E. F. (1975). A spreading-activation theory of semantic processing. Psychological Review, 82, $407-428$. 
Crocker, J., Thompson, L., McGraw, K., \& Ingerman, C. (1987). Downward comparison, prejudice, and evaluations of others: Effects of self-esteem and threat. Journal of Personality and Social Psychology, 52, 907 - 916.

Crocker, J., \& Wolfe, C. T. (2001). Contingencies of self-worth. Psychological Review, 108, $593-623$.

Dunning, D., Leuenberger, A., \& Sherman, D. A. (1995). A new look at motivated influence: Are self-serving theories of success a product of motivational forces? Journal of Personality and Social Psychology, 69, 58 - 68.

Festinger, L. (1954). A theory of social comparison processes. Human Relations, 7, 117 - 140.

Festinger, L. (1957). A theory of cognitive dissonance. Stanford, CA: Stanford University Press.

Gibbons, F., Helweg-Larsen, M., \& Gerrard, M. (1995). Prevalence estimates and adolescent risk behavior: Cross-cultural differences in social influence. Journal of Applied Psychology, 80, $107-121$.

Goethals, G. R., Messick, D. M., \& Allison, S. T. (1991). The uniqueness bias: Studies of constructive social comparison. In J. Suls \& T. A. Wills (Eds.), Social comparison research: Contemporary theory and research (pp. 149 - 176). Hillsdale, NJ: Lawrence Erlbaum Associates, Inc.

Higgins, E. T. (1996). Knowledge activation: Accessibility, applicability, and salience. In E. T. Higgins \& A. W. Kruglanski (Eds.), Social psychology: Handbook of basic principles (pp. 133 - 168). New York, NY: Guilford Press.

Hoyle, R., Kernis, M., Leary, M., \& Baldwin, M. (1999). Selfhood: Identity, esteem, regulation. Boulder, CO: Westview Press.

Hughes, F. M., \& Seta, C. E. (2003). Gender stereotypes: Children's perceptions of future compensatory behavior following violations of gender roles. Sex Roles, 49, $685-691$.

Keppel, G. (1991). Design and analysis (3rd ed.). Englewood Cliffs, NJ: Prentice-Hall.

Kernis, M. H. (Ed.). (1995). Efficacy, agency, and self-esteem. New York, NY: Plenum Press.

Kernis, M. H., \& Waschull, S. B. (1995). The interactive roles of stability and level of selfesteem:

Research and theory. In M. P. Zanna (Ed.), Advances in experimental social psychology (Vol. 27, pp. 93 - 141). San Diego, CA: Academic Press.

Klar, Y., \& Giladi, E. E. (1997). No one in my group can be below the group's average: A robust positivity bias in favor of anonymous peers. Journal of Personality and Social Psychology, 73, 885 - 901.

Klar, Y., \& Giladi, E. E. (1999). Are most people happier than their peers, or are they just happy? Personality and Social Psychology Bulletin, 25, 586 - 595. 
Lockwood, P., \& Kunda, Z. (1997). Superstars and me: Predicting the impact of role models on the self. Journal of Personality and Social Psychology, 73, $91-103$.

Messick, D. M., Bloom, S., Boldizar, J. P., \& Samuelson, C. D. (1985). Why we are fairer than others? Journal of Experimental Social Psychology, 21, 480 - 500.

Mussweiler, T. (2001). "Seek and ye shall find": Antecedents of assimilation and contrast in social comparison. European Journal of Social Psychology, 31, 499 - 509.

Mussweiler, T. (2003). Comparison processes in social judgment: Mechanisms and Consequences. Psychological Review, 110, 472 - 489.

Mussweiler, T., \& Bodenhausen, G. (2002). I know you are but what am I? Self-evaluative consequences of judging in-group and out-group members. Journal of Personality and Social Psychology, 82, $19-32$.

Perloff, L. S., \& Fetzer, B. K. (1986). Self - other judgments and perceived vulnerability to victimization. Journal of Personality and Social Psychology, 50, $502-510$.

Rosenberg, M. (1965). Society and the adolescent self-image. Princeton, NJ: Princeton University Press.

Seta, C. E., Seta, J. J., \& Goodman, R. C. (1998). Social identity orientation and the generation of compensatory expectations: Schema maintenance through compensation. Basic and Applied Social Psychology, 20, 285 - 291.

Seta, J. J., Donaldson, S., \& Seta, C. E. (1999). Self-relevance as a moderator of selfenhancement and self-verification. Journal of Research in Personality, 33, 442 - 462.

Seta, J. J., Hundt, G. M., \& Seta, C. E. (1995). Costs influence on attitudes and value: Beyond dissonance theory. Basic and Applied Social Psychology, 17, 267 - 283.

Seta, J. J., \& Seta, C. E. (1982). Personal equity: An intrapersonal comparator system analysis of reward value. Journal of Personality and Social Psychology, 43, $222-235$.

Seta, J. J., \& Seta, C. E. (1992). Personal equity-comparison theory: An analysis of value and the generation of compensatory and non-compensatory expectations. Basic and Applied Social Psychology, 13, $47-66$.

Seta, J. J., \& Seta, C. E. (1993). Stereotypes and the generation of compensatory and noncompensatory expectancies of group members. Personality and Social Psychology Bulletin, 19, $722-731$.

Seta, J. J., Seta, C. E., \& Erber, M. W. (1993). The role of cost in generating expectations and value: A personal equity comparison theory analysis. Basic and Applied Social Psychology, 14, $103-119$. 
Seta, J. J., Seta, C. E., \& McElroy, T. (2003). Attributions in the service of stereotype maintenance: A schema-maintenance through compensation analysis. Personality and Social Psychology Bulletin, 29, 151 - 163.

Steele, C. M., Spencer, S. J., \& Lynch, M. (1993). Self-image resilience and dissonance: The role of affirmational resources. Journal of Personality and Social Psychology, 64, $885-$ 896.

Strack, F., \& Mussweiler, T. (1997). Explaining the enigmatic anchoring effect: Mechanisms and selective accessibility. Journal of Personality and Social Psychology, 73, 437 - 446.

Suls, J., \& Wills, T. A. (Eds.). (1991). Social comparison research: Contemporary theory and research (pp. 149 - 176). Hillsdale, NJ: Lawrence Erlbaum Associates, Inc.

Swann, W. B., \& Schroeder, D. G. (1995). The search for beauty and truth: A framework for understanding reactions to evaluations. Personality and Social Psychology Bulletin, 21, $1307-1318$.

Tesser, A., Crepaz, N., Collins, J. C., Cornell, D., \& Beach, S. R. H. (2000). Confluence of selfesteem regulation mechanisms: On integrating the self-zoo. Personality and Social Psychology Bulletin, 26, 1476 - 1489.

Weinstein, N. D. (1980). Unrealistic optimism about future life events. Journal of Personality and Social Psychology, 39, 806 - 820.

Wills, T. A. (1981). Downward comparison principles in social psychology. Psychological Bulletin, 90, $245-271$. 